\title{
Variables that Predict Affirmative, Responses to the Item "If God Told Me to Kill I Would Do it in His Name" : Implications for Radical Religious Behaviours
}

\section{Michael A Persinger}

Departments of Psychology and Biology, Laurentian University, Sudbury, Ontario, P3E 2C6, Canada

"Corresponding author: Michael A Persinger, Human Studies, Behavioral Neuroscience and Biomolecular Sciences Programs, Departments of Psychology and Biology, Laurentian University, Sudbury, Ontario, P3E 2C6, Canada, Tel: 7056751151; E-mail: mpersinger@laurentian.ca

Rec date: May 04, 2016; Acc date: May 21, 2016; Pub date: May 26, 2016

Copyright: (C) 2016 Persinger MA. This is an open-access article distributed under the terms of the Creative Commons Attribution License, which permits unrestricted use, distribution, and reproduction in any medium, provided the original author and source are credited

\begin{abstract}
Groups of people who feel sanctioned by God or some equivalent perceived Deity to kill other groups of people who are considered external to the group sometimes constitute armies that eliminate others because they are different. Multiple regression was employed to discern which items from the Personal Philosophy Inventory administered to over 1,200 first year university students over 11 years predicted an affirmative response to the item "If God told me to kill I would do it in His name". About 7\% of the population answered "yes" to this item. Affirmative responders also stated that: they were "special agents of God", people should be guided to ensure spiritual development and they went to church weekly. They also believed a sign would be given when a tough decision was to be made. Individuals who stated they would kill in God's name endorsed more traditional religious beliefs than exotic beliefs. The consistency of the pattern over time for this population of normal young people living in an identified democratic country suggested a potential trait (that if applied over large populations) might produce themecongruent behaviors. Knowing the characteristics of individuals who respond in this manner might facilitate the development of strategies to reduce the dangerous applications of these beliefs and their recruitment by extremists groups.
\end{abstract}

Keywords: Killing in god's name; Religious extremism; University students; Religious beliefs; Exotic beliefs

\section{Introduction}

Intergroup human conflicts often result in mutual group homicides that are justified by the unifying belief exhibited by each group. Lorenz [1] considered the dark side of culture to be the conceptualization of individuals external to the group as being less than human. This belief facilitates the justification for the other group to be subjugated, enslaved, or killed [2]. Historically and cross-culturally the ultimate source of the specialness of a specific group or culture is attributed to a superhuman entity to whom the culture is allegedly accountable and who in turn ensures that individuals within that culture, assuming he or she follows the prescription of behaviors, acquire the maximum maintenance of individual identity: immortality. Most countries still contain constitutional guarantees that the belief in a god that defines a group can neither be challenged nor removed. Through implicit verbal chains such embedded endorsement validates the religious premise and its extrapolations.

If elimination of other groups of people who are different or who are not within the definition of the reference group is coupled with the confirmed accessibility to a person's immortality then the potency of this belief becomes potentially lethal [3]. Given the egocentric nature of human experience and the structural organization of the human brain [4-6], the prospect for immortality and some degree of postdemise continuance of the self might be considered a primal motivation to execute the most extreme minutia of the belief. One would expect if this propensity were a trait or trait-like it should display a normal distribution in any given population with a persistent proportion of occurrence.

Identifying the properties of people who are likely to endorse extreme behaviors, such as killing others if they believe the Deity might condone it, may allow development of strategies to prevent or retard the behavior. Here results are presented that suggest a larger than expected proportion of apparently normal young people in an allegedly democratic and cultural pluralistic country stated they would kill other people if their God told them to kill. The additional items endorsed by these men and women indicate an aggregate of responses that might be useful in inferring who might be prone to recruitment by extreme religious groups.

\section{Methods and Materials}

The yes, no $(0,1)$ responses to the 140 item personal philosophy inventory (PPI) constructed by Persinger and Makarec [7] were obtained from a total of 1,208 university students who were enrolled in a first year Introduction to Psychology course from the years 1982 through 1992 (11 years). The inventory was administered the first day of the fall semester class at the end of a two hour lecture. Most of the items were designed to discern experiences associated with complex partial epileptic-like experiences as well as intrinsic beliefs. The construct validity of the items as well as test-retest reliability of clusters of items has been reported elsewhere [8]. Most (90\%) of the students were between 18 and 19 years of age and single; $42 \%$ of the students were male. Approximately $75 \%$ of the students endorsed a Christian religion (Roman Catholic or various forms of Protestantism) as their primarily affiliation. 
The data had been loaded into electronic data files. Multiple regressions were completed with item 136 "If God told me to kill I would do it in His name" as the dependent variable. All of the other variables were included in the independent variable list. In order to minimize trivial effects the maximum numbers of steps allowed for the regression was set $=5$. Additional analyses of variance, polynomial trends, and mixed design (within and between subject variables) were completed by PC SPSS16 (Statistical Package for Social Scientists).

\section{Results}

The (first five) variables that entered the multiple regression analysis that predicted the answers by individuals to other items who responded "yes" vs "no" to the Item 136: "If God told me to kill I would do it in His name" were intrinsically consistent as an aggregate even when subsets of the population were analyzed separately. Their identification as well as the partial slopes (B) and standard errors are indicated in parentheses. All $t$-statistics for these variables were $t>3.50$ and statistically significant $(\mathrm{p}<0.001)$. The variables that entered as indicated by their step sequence were Item $129(0.101,0.025)$ "I am a special agent of God", Item 128 (0.059, 0.015) "Most people should be guided to ensure their spiritual development", Item $50(0.057,0.017)$ "I go to church at least once a week most months", item $40(0.068,0.020)$ "When I have a tough decision to make a sign will be given and I will know what to do" and Item $16(-0.141,0.041)$ "Once in a while I put off until tomorrow what I should do today". The latter was a negative slope. The constant was 0.146 .

The percentage of 1,208 respondents who indicated affirmative responses to each item were: Item 136: 7\%, Item 129: 10\%, Item 128: 41\%, Item 50: $28 \%$, Item 40: $16 \%$ and Item 16: $97 \%$. The multiple $\mathrm{r}$ value was $0.29[\mathrm{~F}(5,1203)=22.74, \mathrm{p}<0.001]$; the standard error of the estimate was 0.245 . Approximately $8 \%$ of the variance (adjusted $\mathrm{r}^{2}$ ) in the response profile to item 136 was accommodated by the combination of these 5 variables. Post hoc addition of other variables by increasing the number of steps up to 10 did not appreciably alter the magnitude of the association.

To discern if there had been a shift in responding over the 11 years (1982-1992) of the data collection, polynomial analyses were completed for each of the six variables (the dependent and the 5 independent items) as a function of decade. There was no statistically significant change in the proportion of yes responses to Item 136 $(\mathrm{F}=1.43)$, Item $129(\mathrm{~F}=0.72)$ or Item $40(\mathrm{~F}=0.44)$ as a function of year. However there was a statistically significant linear diminishment of affirmative responses over time for item $50(\mathrm{~F}=21.79)$ from 35\%-18\%. Item 128 decreased $(\mathrm{F}=21.79)$ from $50 \%-34 \%$.

To discern if traditional items that constitute beliefs differentiated the populations who responded "yes" or "no" to the key item, a threeway analysis of variance with one within level (two types of belief clusters) and two between levels (no, yes to item 136) and gender was completed to isolate the specificity of the effect. The two belief clusters were each constructed of five items. For the traditional belief the items were: Item 126: "I believe in the second coming of Christ", Item 26: "I believe there is a God", Item 80: "Everything is turning out just like the prophets in the Bible said it would", Item 128: "Most people should be guided to ensure their spiritual development", and Item 43: "God has healed me or one of my family members on at least one occasion". For the exotic beliefs the items include Item 104: "Although I am not sure, there is a good possibility that I have lived in a previous time", Item 115: "Alien intelligence is probably responsible for UFOs", Item 44: "I would like to time travel", Item 94: “Telepathy (ESP) is a real phenomenon", and Item 46: (negatively weighted): “There are no such things as ghosts or haunts", i.e. there are ghosts and haunts. The means and standard deviations for the two clusters of items were: traditional: $46 \%$ (29\%) and exotic: $52 \%(27 \%)$.

The results of the three way analysis of variance indicated a statistically significant interaction $[\mathrm{F}(1,1207)=26.81, \mathrm{p}<0.001 ; 2 \%$ of variance] between the type of belief and the response to the item that the person would kill in God's name. Post hoc analyses indicated there was no statistically significant difference in the endorsement proportion for exotic beliefs by those who reported they would ( $M=54 \%, S D=27 \%)$ or would not kill (52\%, SD=27\%).

However those who said they would kill in God's name if he told them to kill endorsed significantly more traditional belief items in the cluster $(\mathrm{M}=68 \%, \mathrm{SD}=24 \%)$ then those who said they would not kill $(\mathrm{M}=44 \%, \mathrm{SD}=29 \%)$. There was a weaker $[\mathrm{F}(1,1207)=4.67, \mathrm{p}<0.05]$ but statistically significant interaction between gender and type of belief. Men who reported they would kill displayed higher scores for traditional beliefs compared to women. However there was no statistically significant main effect between genders $[\mathrm{F}(1,1207)=2.75$, $\mathrm{p}>0.05$ ] for proportions of endorsed beliefs for either the traditional or exotic cluster.

\section{Posthoc Analyses}

Differentiation of which personality or behavioral patterns are associated with the propensity to endorse Item 136 was pursued by closely examining a subset of 80 subjects for which data from the MMPI-168 (Minnesota Multiphasic Personality Inventory) and Castell's 16 PF (Personality Factor) questionnaires had been collected separately. Multiple regressions with the item 136 as the dependent were completed for each inventory separately. Whereas none of the factors from the $16 \mathrm{PF}$ entered the equation the scores for the Depression and Psych asthenia scales from the MMPI entered the equation to predict a yes response to item 136. The combination of increased depression scale scores and diminished psych asthenia scaled scores was associated with a multiple $\mathrm{r}$ of $0.41 \mathrm{~F}(1,79)=7.88, \mathrm{p}<0.01$ ] with positive endorsement of killing in God's name.

\section{Discussion}

A person's response to a questionnaire does not completely accommodate the realistic contexts associated with social pressure and cultural conditioning. They determine a substantial proportion of beliefs and attitudes. However profiles from questionnaire studies obtained from relatively large populations can be considered a marker or indicator of the characteristics of the population within specific conditions. In the present population of young first year university students, primarily Roman Catholic, from an identified democratic country about 7\% stated they would kill in God's name if he told them to do so. Even if only 1 in 10 of those students who answered affirmatively would translate this response to an actual behavior, the absolute size of that group within a population of 100 million people would about 700,000. These are sufficient numbers for the foundation of a formidable army of individuals who could engage in extreme behaviors.

If the results obtained in this study can be generalized to other cultural groups people who say they would kill if a god told them are more likely to endorse items that: 1) they are special agents of god, 2) people should be guided to ensure their spiritual development, 3) they 
depend upon intuition for difficult decisions, 4) they attend church or the equivalent weekly and, and 5) they are enthusiastic in their executions of tasks, i.e. they do not procrastinate. The absence of abnormal behaviors and eccentric classic personality differences from the reference population, as indicated by the absence of statistically significant differences for the primary 16 factors of personality and even the psychiatric-based inventory (MMPI), is expected. Extreme beliefs are not necessarily indicators of abnormal behaviors or psychiatric anomalies. The only minor difference was for two scales by which depression and "ruminative" anxiety are inferred. People who endorsed the killing of others in God's name exhibited a combination of mildly diminished ruminative anxiety and more than typical depression. This is not a frequent combination.

The percentage of people in this transition from high school to university that endorsed the question "If God told me to kill, I would do it in his Name" did not change over the years of measurement. For the questionnaire method of behavioral inferences this consistency over time usually suggests a relatively stable trait that is not affected by social nuances or change. It is not clear if the people who responded affirmatively to this item would change as they aged. What is clear is that the proportion of young people (primarily between the ages of 18 and 20 years and primarily men) who stated they would kill others in God's name remained relatively constant. This is a peak recruiting age for most condoned and non-condoned armies in many cultures.

The significance of beliefs in mediating or mitigating behavior can be considered a fundamental principle of sociological phenomena. Persinger and Makarec [9] showed that when exotic and traditional beliefs were employed as within subject factors in a between factor design that involved groups of people who reported they had or had not had a religious experience and who either did or did not attend church weekly, the proportions of average beliefs were comparable. The revealing feature was the interaction between type of belief and the church attendance-related behaviors. Those that endorsed more proportions of traditional beliefs endorsed fewer exotic beliefs and vice versa. One interpretation is that people believe in a more or less a persistent manner and if the endorsement of one type of belief is elevated the adherence to other types of beliefs is diminished.

Studying groups of people with items such as those discussed in this analysis may reveal the factors that influence life-changing decisions such as joining organizations that allow the belief to be manifested. One of the most compelling results of my studies in this domain over the last four decades has been the realization that university education allows the person to acquire technical expertise. It rarely changes the core or structural belief configuration of the person. This would suggest that the person after university training has more potential technology to be applied in the support and execution of beliefs.

The prevalent social climate of censorship of individuals who challenge religious belief systems may be indirectly facilitating a future condition whereby belief-condoned behaviors can never be challenged because the act would be considered offensive and even nonconstitutional to the local culture. There are event prohibitive boundaries appearing within the classrooms of universities and colleges. Professors who employ critical thinking and discuss the full range of possibilities and veridicalities are removed from the classroom or dismissed from the institution. The pretense is to avoid offending the delicate nature of consumers which is the contemporary administrative business label for students in the transition of salesbased education.
Concomitant biases of interpretation have been shown experimentally. Persinger [10] extracted pairs of quotes that involved god-condoned killing of others from religious books that included: The Bible, The Koran, The Book of Mormon, The Tibetan Book of the Dead, and The Egyptian Book of the Dead. Half of the numbers of the subject population was told the quotes were from psychiatric patients. The responsibility of the subjects was to rank which patients were most dangerous with respect to being released or not released from care. The other half of the student population was given the same task and same quotes but the source of the quotes were identified. The group who did not know the source of the quotes ranked the statements (from alleged patients) from The Bible and The Koran as more dangerous compared to those who knew the source. In other words those subjects who were evaluating the "dangerousness" of statements and knew they were sanctioned by religious beliefs or traditions rated the content differently when the source was known. One interpretation is that the nuances of political correctness and government interventions, such as variants of "respectful work place" policies that encourage censorship of free expression, attenuated the critical thinking that would have otherwise assessed an enhanced degree of dangerousness [11-13].

\section{Acknowledgement}

This research is dedicated to my Young Colleague Dr. Rob Buckman who left us far too soon.

\section{References}

1. Lorenz K (1963) On aggression. Methuen: Austria

2. Persinger MA (1987) Neuropsychological bases of God beliefs. Plenum Press, NewYork, USA.

3. Persinger MA (1985) Death anxiety as a semantic conditioned suppression paradigm. Perceptual and Motor Skills 60: 827-830.

4. Hayward RD, Owen AD, Koenig HG, Steffens DC, Payne ME (2011) Associations of religious behavior and experiences with extent of regional atrophy in the orbitofrontal cortex during older adulthood. Religion, Brain and Behavior 1: 103-118.

5. Persinger MA (2009) Are our brains structured to avoid refutations of the belief in God? An experimental study. Religion 39: 34-42.

6. Wuerfel J, Krishnamoorthy ES, Brown RJ, Lemieux L, Koepp M, et al. (2016) Religiosity is associated with hippocampal but not amygdala volumes in patients with refractory epilepsy. Journal of Neurology, Neurosurgery and Psychiatry 75: 640-642.

7. Persinger MA, Makarec K (1993) Complex partial epileptic-like signs as a continuum from normal to epileptics: normative data and clinical populations. J Clin Psychol 49: 33-45.

8. Makarec K, Persinger MA (1990) Electroencephalographic validation of a temporal lobe signs inventory in a normal population. J Res Pers 24: 323-327.

9. Persinger MA, Makarec K (1990) Exotic beliefs may be substitutes for religious beliefs. Perceptual and Motor Skills 71: 16-18.

10. Persinger MA (2004) Perceived dangerousness to public safety of paraphrases from the Koran, Old Testament, Book of Mormon, Tibetan Book of the Dead, and Egyptian Book of the Dead. Perceptual and Motor Skills 98: 1345-1355.

11. Fingelkurts AA (2009) Is our brain hardwired to produce God, or is our brain hardwired to perceive God? A systematic review on the role of the brain in mediating religious experience. Cognitive Processes 10: 293-326.

12. Persinger MA (1997) I would kill in God's name: role of sex, weekly church attendance, report of a religious experience and limbic liability. Perceptual and Motor Skills 85: 128-130.

13. Buckman R (2002) Can we be good without God? Biology, behavior and the need to believe. Prometheus: (Amherst) NewYork, USA. 